\title{
On the influence of a spatial grid structure on numerical simulation of a shock wave in a flow around 3D model
}

\author{
I.A. Shirokov ${ }^{1}$, T.G. Elizarova ${ }^{2}$ \\ ${ }^{1}$ Moscow State University, Faculty of Computational Mathematics and Cybernetics, \\ Moscow, 119991, Russia \\ ${ }^{2}$ Keldysh Institute of Applied Mathematics, \\ Moscow, 125047, Russia \\ ivanshirokov@inbox.ru
}

\begin{abstract}
The influence of 3D spatial grid structure on a quality of the numerical modeling of viscous compressible gas flow at Mach number equal to 3 near a forward-facing part of a rectangular cylinder is demonstrated. The obtained results show that the grid structure produces critical effects on the shape and the intensity of the head shock wave, which can exceed the influence of other numerical effects. The calculations are performed using a finite volume method implemented for regularized or quasi-gasdynamic equations.
\end{abstract}

Keywords: shock wave modeling, quasi-gas-dynamic algorithm, grid structure.

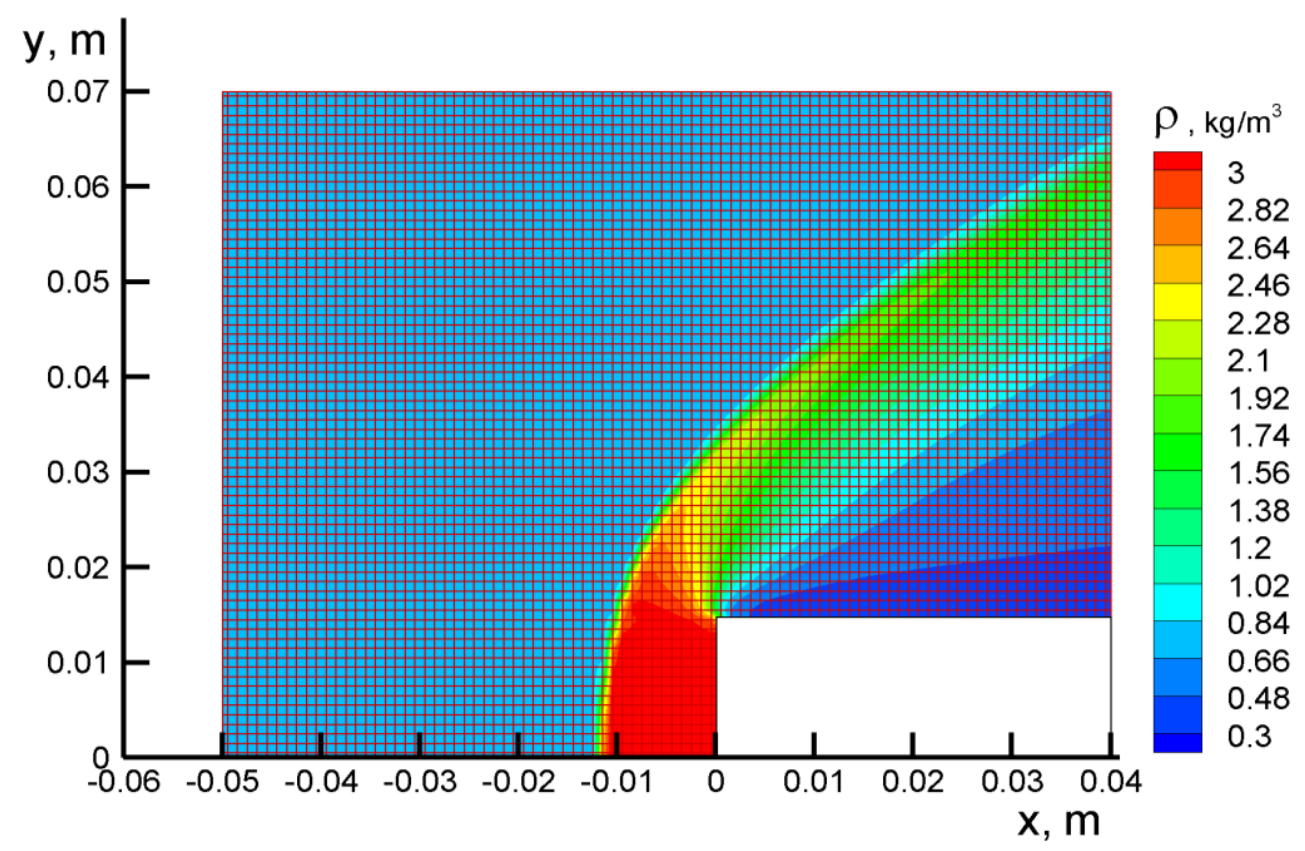

QGD-based modeling of the supersonic flow: density contours and the grid structure 


\title{
О влиянии структуры пространственной сетки на результаты численного моделирования ударной волны в задаче обтекания трехмерной модели
}

\author{
И.А.Широков ${ }^{1}$, Т.Г. Елизарова ${ }^{2}$ \\ ${ }^{1}$ Московский государственный университет им. М.В. Ломоносова, $\phi-m$ ВМК, \\ Россия, Москва, 119991, ГСП-1, Ленинские горы, д. 1, стр. 52 \\ ${ }^{2}$ Институт прикладной математики им. М.В. Келдыма РАН, \\ Россия, Москва, 125047, Миусская пл. 4а \\ ivanshirokov@inbox.ru
}

\begin{abstract}
Аннотация
Демонстрируется влияние структуры трехмерной пространственной сетки на качество численного решения в задаче об обтекании торцевой части прямоугольного параллелепипеда потоком вязкого сжимаемого газа при числе Маха, равном 3. Из полученных результатов следует, что структура сетки оказывает определяющее влияние на форму и интенсивность головной ударной волны, которое может превосходить влияние других сеточных эффектов. Расчеты выполнены с применением метода конечного объема, выписанного для регуляризованных, или квазигазодинамических, уравнений.
\end{abstract}

Ключевые слова: моделирование ударных волн, квазигазодинамический алгоритм, структура вычислительной сетки.

\section{1. Введение}

Вопросы точности численного решения задач аэродинамики сверхзвукового обтекания моделей имеют первостепенное значение для проектирования и исследования полетных характеристик реальных аппаратов. Точность численных алгоритмов обусловлена многими факторами. К ним, в первую очередь, относятся порядок точности самой разностной схемы как по времени, так и по пространству, а также структура искусственных добавок и лимитеров, обеспечивающих ее монотонность, количество пространственных узлов разностной сетки и наконец, структура самой сетки.

Для численного моделирования обтекания аппаратов сложной структуры неизбежно использование сложных многокомпонентных неструктурированных сеток, к которым относятся тетраэдральные сетки. Качество расчета в целом, помимо характеристик разностного алгоритма, в значительной мере определяется качеством используемой пространственной сетки. При этом трудоемкость создания сеток хорошего качества бывает сравнима с трудоемкостью всего расчета в целом, и задача построения «хороших» пространственных сеток приобретает первостепенное значение [1,3].

В данной работе на примере классической задачи о внешнем трехмерном сверхзвуковом обтекании торцевой части параллелепипеда показана роль пространственной сетки и ее влияние на точность моделирования головной ударной волны при решении полных уравнений Навье - Стокса для больших чисел Рейнольдса. В качестве метода численного решения применяется алгоритм, основанный на использовании регуляризованных, или квазигазодинамических (КГД) уравнений. 


\section{2. Постановка задачи}

Рассмотрим модель в форме прямоугольного параллелепипеда, поперечные размеры которого в декартовой системе координат определяются как $-0.015<y<0.015$, $-0.015<z<0.015$ (здесь и ниже размеры приведены в метрах). Модель расположена с нулевым углом атаки по отношению к набегающему потоку. Расчетная область представляет собой прямоугольный параллелепипед, внутри которого расположена модель в набегающем потоке воздуха, направленном вдоль оси $x$. Параметры набегающего потока имеют следующие значения: газовая постоянная $\mathrm{R}=287$ Дж/(кг·K), показатель адиабаты $\gamma=1.4$, число Прандтля $\operatorname{Pr}=14 / 19$, показатель межмолекулярного взаимодействия $\omega=0.74$. Рассматривается число Маха $\mathrm{Ma}=3$. Число Рейнольдса, отнесенное к 1 м, равняется $\operatorname{Re}=4.7 \times 10^{7}$.

В работе [4] экспериментально и численно исследуется внешнее обтекание осесимметричного тела. Результаты, полученные в упомянутой работе, представляют большой интерес как эталонные данные, которые могут быть использованы при разработке и тестировании численных методов, предназначенных для моделирования газодинамических течений. В связи с этим размеры модели и безразмерные параметры набегающего потока в данной работе, приведенные в предыдущем параграфе, выбраны такими же, как в [4].

Для определения размерных параметров набегающего потока воспользуемся данными стандартной атмосферы. Пусть рассматриваемая модель движется в атмосфере на некоторой высоте. Тогда при заданном числе Маха и заданном характерном размере можно построить зависимость числа Рейнольдса от высоты полета в атмосфере, пользуясь данными стандартной атмосферы (ГОСТ 4401-81). На рис. 1 приведена такая зависимость $\operatorname{Re}$ от высоты полета для $\mathrm{Ma}=3$. Поскольку изображенная зависимость имеет монотонный характер, высота полета определяется однозначно. Зная высоту, можно определить размерные параметры набегающего потока в стандартной атмосфере.

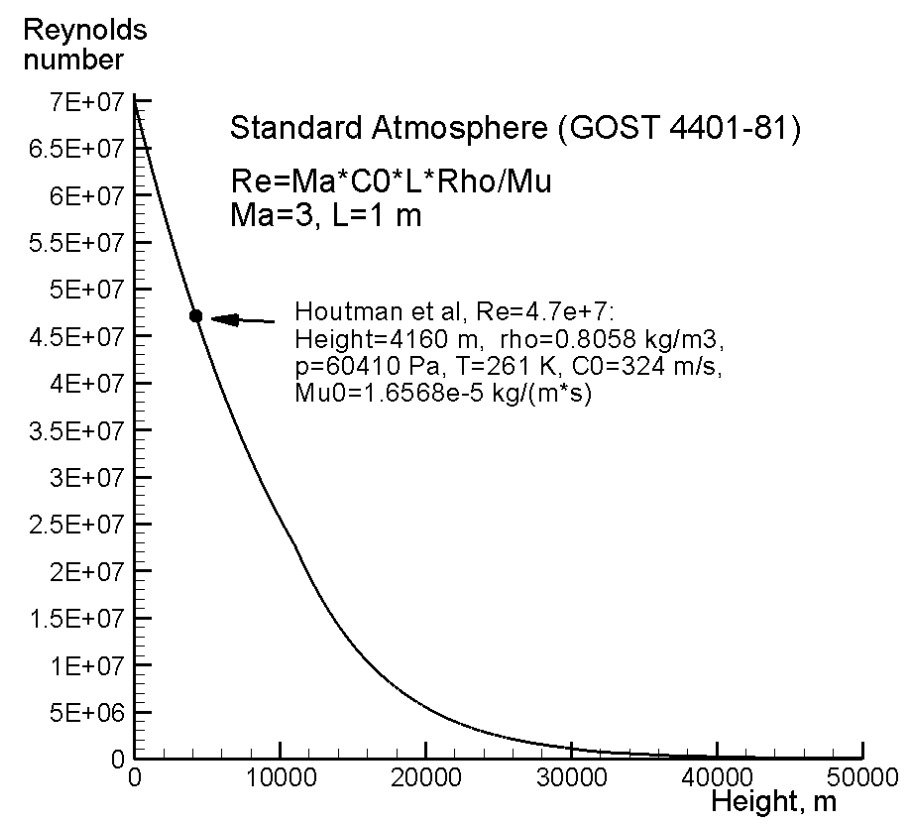

Рис. 1. Зависимость числа Рейнольдса от высоты полета

Полученные описанным способом параметры набегающего потока имеют следующие значения: высота полета $4160 \mathrm{м}$, плотность $\rho_{0}=0.8058 \kappa г / \mathrm{m}^{3}$, давление $p_{0}=60410$ Па, температура $T_{0}=261 \mathrm{~K}$, скорость звука $c_{0}=324 \mathrm{M} / \mathrm{c}$, коэффициент динамической вязкости $\mu_{0}=1.6568 \times 10^{-5} \mathrm{\kappa} /(\mathrm{M} \cdot \mathrm{c})$. 


\section{3. Математическая модель}

В качестве метода численного решения макроскопических уравнений газодинамики применяется алгоритм, основанный на использовании регуляризованных, или квазигазодинамических (КГД) уравнений. Способ построения этих уравнений и их применение к задачам аэродинамики описаны, в частности, в [5-10]. Эта система может рассматриваться как регуляризованный вид системы уравнений Навье-Стокса (HC), который получается путем естественной процедуры усреднения исходной системы уравнений газовой динамики на малом пространственно-временном интервале, что приводит к сглаживанию (регуляризации) исходной системы уравнений и выражается в появлении в ней добавок, пропорциональных малому параметру с размерностью времени. Последнее позволяет использовать для их численной реализации конечно-объемные алгоритмы с аппроксимацией всех потоковых величин центральными разностями без использования лимитеров для обеспечения их монотонности и условной устойчивости.

Таким образом, построенные на основе КГД уравнений разностные схемы имеют ряд аналогий со схемами Годунова, к основным достоинствам которых относятся [11]: однородность и наглядность численного алгоритма, монотонность решения и выполнение закона о неубывании полной энтропии, а также возможность моделирования разрывных решений. К основному недостатку этого подхода относят его первый порядок точности по пространству. Имеется целый ряд работ, посвященных повышению порядка точности схем Годунова, однако повышение порядка точности по пространству уже не гарантирует выполнение условия неубывания полной энтропии. А это, в свою очередь, может приводить к появлению нефизичных решений уравнений Эйлера. В частности, в [11] показано, что в зависимости от величины схемной вязкости и начальных условий могут формироваться как традиционно известные стационарные картины течения, так и нефизичные нестационарные картины с наличием в них косых ударных волн. В природе такие решения выбраковываются за счет наличия вязких эффектов, которые обязательно присутствуют в течении. В отличие от схем Годунова, повышение порядка КГД схем не проводилось, и в рамках КГД подхода таких нефизичных картин течения не наблюдалось.

В декартовых координатах в отсутствии внешних сил и источников тепла КГД система может быть представлена в виде [6]

$$
\begin{gathered}
\frac{\partial}{\partial t} \rho+\nabla_{i} j_{m}^{i}=0, \\
\frac{\partial}{\partial t} \rho u^{j}+\nabla_{i}\left(j_{m}^{i} u^{j}\right)+\nabla^{j} p=\nabla_{i} \Pi^{i j}, \\
\frac{\partial}{\partial t} E+\nabla_{i}\left(j_{m}^{i} H\right)+\nabla_{i} q^{i}=\nabla_{i}\left(\Pi^{i j} u_{j}\right)
\end{gathered}
$$

Здесь $\rho$ - плотность газа; $u^{i}$ - компоненты его макроскопической скорости; $p$ - давление. Полная энергия единицы объема $E$ и полная удельная энтальпия $H$ идеального политропного газа с показателем адиабаты $\gamma$ вычисляются по формулам

$$
E=\frac{\rho u^{2}}{2}+\frac{p}{\gamma-1}, \quad H=\frac{E+p}{\rho}
$$

Вектор плотности потока массы $j_{m}^{i}$ определяется в виде

$$
j_{m}^{i}=\rho\left(u^{i}-w^{i}\right), \quad w^{i}=\frac{\tau}{\rho}\left(\nabla_{j} \rho u^{i} u^{j}+\nabla^{i} p\right)
$$


Выражения для тензора вязких напряжений $\Pi^{i j}$ и теплового потока $q^{i}$ записываются как

$$
\begin{gathered}
\Pi^{i j}=\Pi_{N S}^{i j}+\tau u^{i} \rho\left(u_{k} \nabla^{k} u^{j}+\frac{1}{\rho} \nabla_{j} p\right)+\tau \delta^{i j}\left(u_{k} \nabla^{k} p+\gamma p \nabla^{k} u_{k}\right), \\
\Pi_{N S}^{i j}=\mu\left(\nabla^{i} u^{j}+\nabla^{j} u^{i}-\frac{2}{3} \nabla^{k} u_{k}\right)+\varsigma \delta^{i j} \nabla^{k} u_{k} \\
q^{i}=q_{N S}^{i}-\tau u^{i} \rho\left(u_{j} \nabla^{j} \varepsilon+p u_{j} \nabla^{j} \frac{1}{\rho}\right), \quad q_{N S}^{i}=-\kappa \nabla^{i} T
\end{gathered}
$$

Здесь $\varepsilon=p / \rho(\gamma-1)$ - внутренняя энергия единицы массы газа; $\Pi_{N S}^{i j}$ и $q_{N S}^{i}-$ тензор вязких напряжений и тепловой поток в системе $\mathrm{HC} ; \mu, \varsigma$ и $\kappa$ - коэффициенты сдвиговой и объемной вязкости и теплопроводности соответственно; $T$ - температура газа. Коэффициент сдвиговой вязкости $\mu$ определим через температурную зависимость [6]

$$
\mu=\mu_{0}\left(\frac{T}{T_{0}}\right)^{\omega},
$$

где $\mu_{0}$ - вязкость газа при температуре $T_{0} ; 0<\omega<1$ - показатель межмолекулярного взаимодействия. Коэффициент теплопроводности вычисляется как

$$
\kappa=\frac{\mu}{\operatorname{Pr}(\gamma-1)}
$$

Коэффициент $\tau$, определяющий дополнительную диссипацию в КГД алгоритме, для вязкого политропного газа имеет порядок характерного времени между столкновениями частиц газа [5-7]. Его величина связывается с коэффициентом сдвиговой вязкости. Для обеспечения устойчивости КГД алгоритма при моделировании сверхзвуковых течений плотных газов выражение для коэффициента $\tau$ модифицируется путем включения в него слагаемого, зависящего от шага пространственной сетки и локальных параметров течения

$$
\tau=\frac{\mu}{p \mathrm{Sc}}+\frac{\alpha h}{c}
$$

где $h$ - характерный размер пространственной ячейки; $c$ - локальная скорость звука; $\alpha-$ настроечный параметр, который в большинстве случаев полагается постоянным числом порядка 1; Sc - число Шмидта, которое для газов близко к единице [5-7].

Коэффициент объемной вязкости может быть вычислен на основе аппроксимационной формулы [6], при этом для стабилизации численного алгоритма вводится искусственная добавка и коэффициент объемной вязкости выражается так

$$
\varsigma=\mu\left(\frac{5}{3}-\gamma\right)+\delta\left(\frac{h}{c}\right) p
$$

Величина регуляризирующей добавки здесь также определяется локальными параметрами и зависит от настроечного коэффициента $\delta$. Такое введение искусственной диссипации в КГД уравнения впервые использовано в $[9,10]$. 


\section{4. Реализация КГД алгоритма}

Для исследования влияния формы ячеек на результат моделирования используются четыре расчетные сетки: регулярная равномерная (1), регулярная неравномерная (2), нерегулярная тетраэдральная (3) и регулярная неравномерная со сгущением (4).

Равномерная сетка (1) имеет кубические ячейки со стороной 1 мм. Расчётная область при этом определяется как $-0.07<y<0.07,-0.07<z<0.07,-0.05<x<0.04$ (размеры здесь и ниже приведены в метрах, если не указано другое). Общее число узлов регулярной сетки (1) 1855088 .

Неравномерная сетка (2) имеет ячейки в виде прямоугольных параллелепипедов. На поверхности модели ячейки имеют такой же шаг 1 мм, как и сетка (1). При удалении от поверхности вдоль каждой оси шаг сетки увеличивается пропорционально $\left(1-\cos \left(l / l_{0}\right)\right)$, где $l$ - расстояние от поверхности модели до текущей ячейки, $l_{0}$ - расстояние от поверхности модели до границы расчетной области в случае сетки (1). Расчетная область в этом случае определяется как $-0.1<y<0.1,-0.1<z<0.1,-0.075<x<0.04$. Неравномерная сетка остается симметричной относительно плоскостей $y=0$ и $z=0$. Общее число узлов неравномерной сетки (2) 363888.

Нерегулярная тетраэдральная сетка (3) построена с помощью библиотеки TetGen [12]. Геометрия модели и расчетной области описывается в файле формата .poly. После построения poly-файла генерация сетки осуществляется по команде tetgen.exe -pq1.4/17a0.00007Y, при этом сетка на поверхности модели, заданная в poly-файле, служит основой построения поверхностных граней тетраэдральных ячеек. Эти грани являются одинаковыми равносторонними прямоугольными треугольниками с длиной катета 0.5 мм. Расчетная область при этом определяется как $-0.07<y<0.07,-0.07<z<0.07,-0.05<x<0.18$. В этом случае модель находится целиком внутри расчетной области, и определяется как $-0.015<y<0.015$, $-0.015<z<0.015,0<x<0.127$. Общее число узлов нерегулярной сетки 153126 , число тетраэдральных элементов 768152 .

Неравномерная сетка (4) имеет ячейки в виде прямоугольных параллелепипедов, как и (2), и строится аналогично (2) с той разницей, что на торце модели ячейки имеют размер 0.3 мм вдоль оси $x$, а на боковой поверхности модели 0.4 мм вдоль направления, перпендикулярного боковой поверхности. Размер других сторон пристеночных ячеек остается равным 1 мм. Расчетная область в этом случае определяется как $-0.1<y<0.1$, $-0.079<z<0.079,-0.043<x<0.04$, сетка остается симметричной относительно плоскостей $y=0$ и $z=0$. Общее число узлов сетки (4), как и (1), 1855088.

На регулярных сетках (1), (2), (4) КГД уравнения аппроксимируются центральными разностями. На равномерной сетке (1) аппроксимация имеет второй порядок точности, в случае неравномерных сеток первый порядок точности. Отметим, что в последнем случае в формулах (11) и (12) шаг по пространству берется постоянным и равным минимальному значению на сетке.

Для расчетов на нерегулярной тетраэдральной сетке (3) применялся доработанный программный комплекс [13], позволяющий проводить расчеты нестационарных вязких газодинамических течений для тел произвольной формы. Значения газодинамических параметров определяются в узлах сетки. Значения газодинамических переменных в точках расчетной области, расположенных между узлами сетки, определяются как среднее арифметическое значений в узлах. Конечно-разностная аппроксимация макроскопических КГД уравнений строится методом контрольных объемов. Барицентрический контрольный объем строится вокруг каждого узла сетки.

Поскольку диссипативные коэффициенты (11) и (12) зависят от локальных параметров, на тетраэдральной сетке алгоритм имеет первый порядок аппроксимации по пространству. 
Для всех сеток решение начально-краевой задачи для сеточных аналогов КГД уравнений находится по явной по времени конечно-разностной схеме, имеющий первый порядок аппроксимации по времени. Расчеты проводились на многопроцессорном комплексе К-100 [14].

\section{5. Результаты моделирования}

На рис. 2-5 представлены результаты, полученные на равномерной сетке (1), слева, и на неравномерной сетке (2), справа. За исключением сетки, остальные параметры расчетов одни и те же: $\alpha=1$, при вычислении коэффициента $\delta$ использовалось следующая зависимость от локального числа Маха: при Ма $\geq 2 \delta=\delta_{1}=0.5$, при Мa $\leq 1.5 \delta=\delta_{2}=1$, в промежутке между значениями числа Маха 1.5 и 2 коэффициент $\delta$ линейно возрастает с числом Maxa [8].

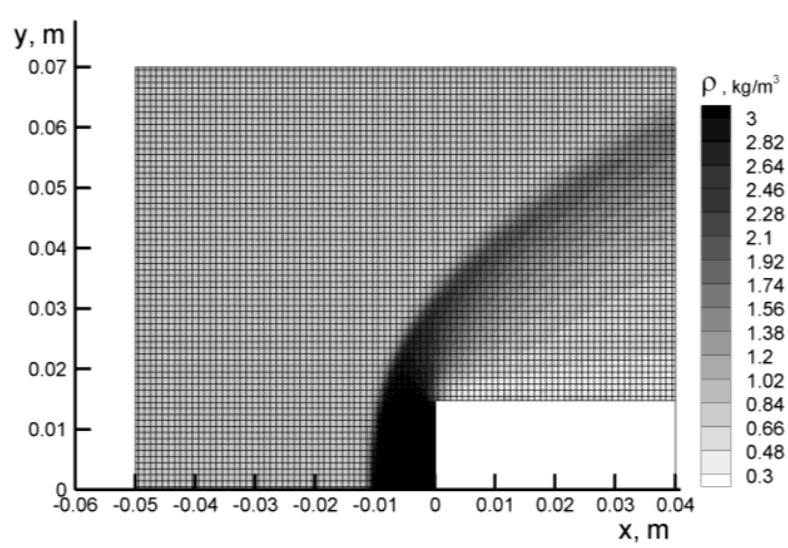

Рис. 2. Равномерная сетка (1)

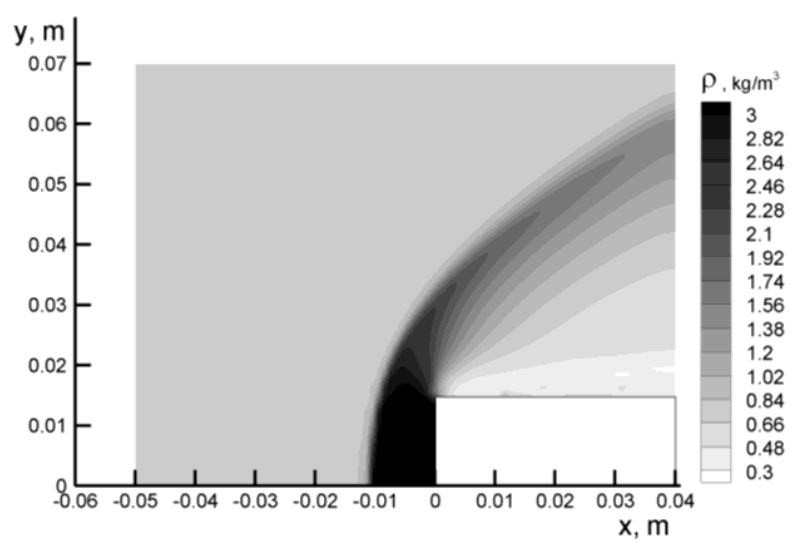

Рис. 4. Равномерная сетка (1)

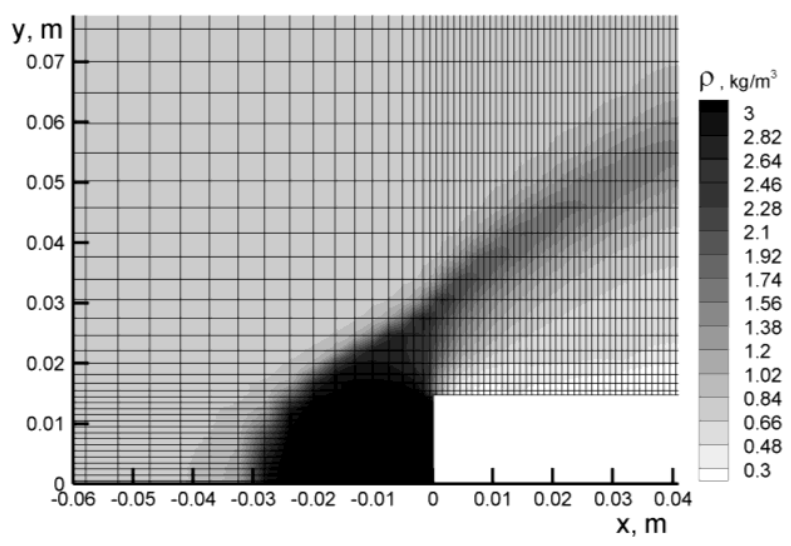

Рис. 3. Неравномерная сетка (2)

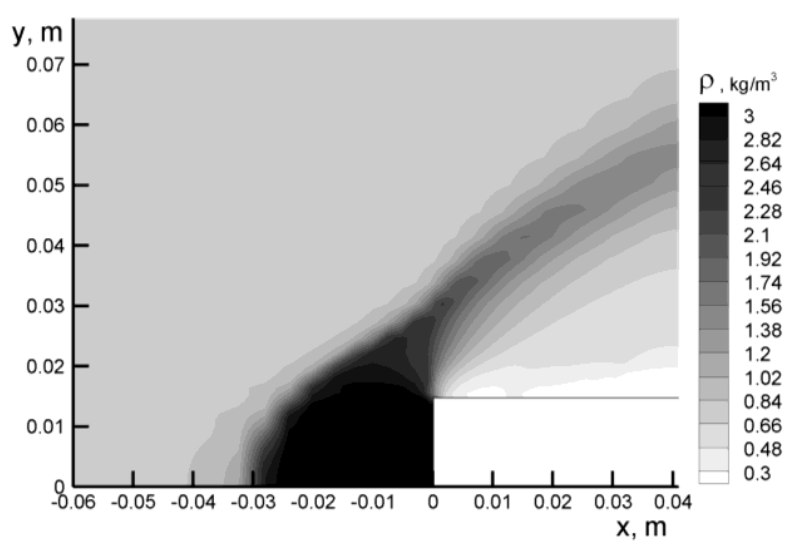

Рис. 5. Неравномерная сетка (2)

На рис. 2 и 3 показана расчетная сетка и линии уровня плотности. На рис. 4 и 5 приведены только уровни плотности, чтобы нагляднее представить отличия в результатах моделирования.

Использование равномерной сетки (рис. 2 и 4) позволяет получить общую картину течения, хорошо соответствующую результатам численного исследования обтекания торца цилиндра, полученным в [6]. Фронт ударной волны достаточно хорошо разрешается и занимает 3-4 ячейки сетки. В случае неравномерной сетки, как видно на рис. 3 и 5, область фронта ударной волны сильно искажена по сравнению с рис. 2 и 4. Фронт головной удар- 
ной волны растянут вдоль направления растяжения ячеек сетки (оси $x$ ), но занимает 4 ячейки, как и в случае равномерной сетки.

Заметим, что, несмотря на наличие сильно искаженного, вытянутого фронта ударной волны (см. рис. 3 и 5), в отличие от схемы Колгана [11], в расчетах по КГД алгоритму нестационарные картины течения с наличием наклонных ударных волн не формируются.

Растяжение ячеек сетки вдоль оси $Y$ (в верхней правой части рисунка) также приводит к искажению формы фронта отходящей ударной волны, хотя и в меньшей степени, чем в случае головной ударной волны, поскольку интенсивность ударной волны здесь ниже.

Вблизи боковой поверхности модели (в районе $x=0.03 \mathrm{M}, y=0.03 \mathrm{м}$, где скорость потока невелика) неравномерная сетка мало искажает результаты моделирования, по сравнению с областью ударной волны.

На рис. 6-9 представлены результаты, полученные на регулярной равномерной сетке (1), слева, и на нерегулярной тетраэдральной (3), справа. Параметры расчетов одинаковы и имеют те же значения, что и для расчетов, представленных на рис. $2-5$, за следующим исключением: коэффициенты искусственной вязкости $\delta_{1}=2$ и $\delta_{2}=5$, то есть выше, чем в предыдущем случае.

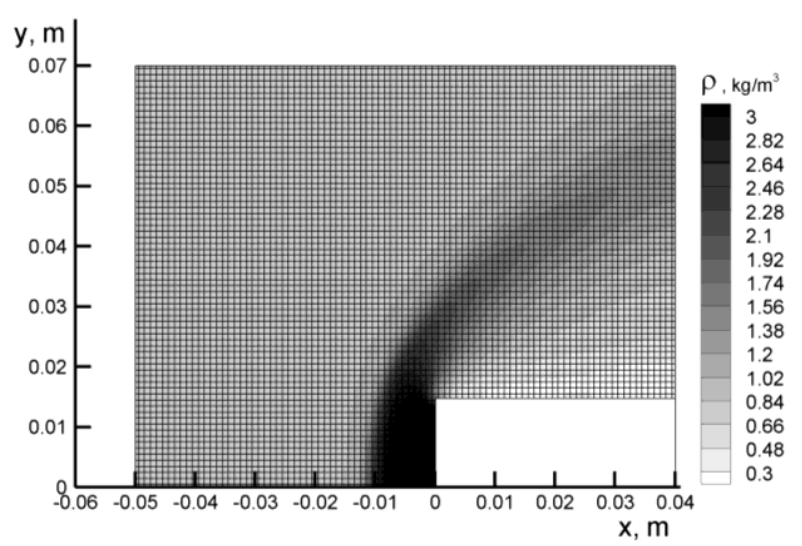

Рис. 6. Равномерная сетка (1)

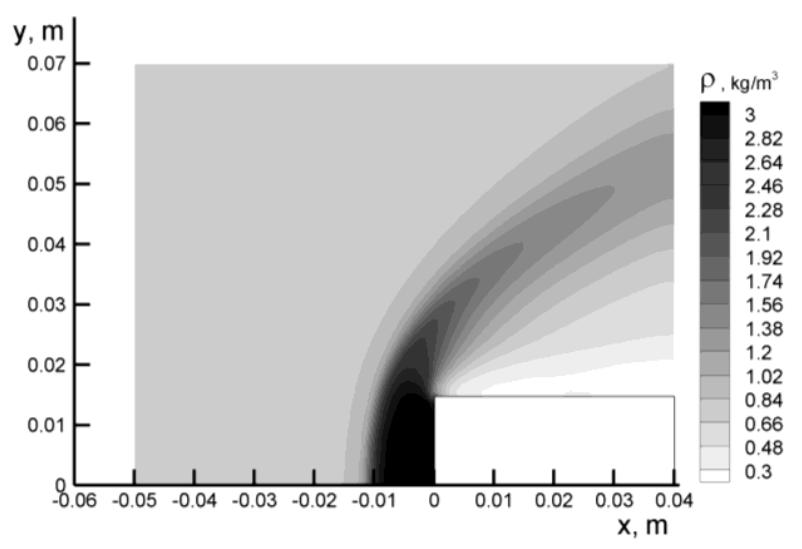

Рис. 8. Равномерная сетка (1)

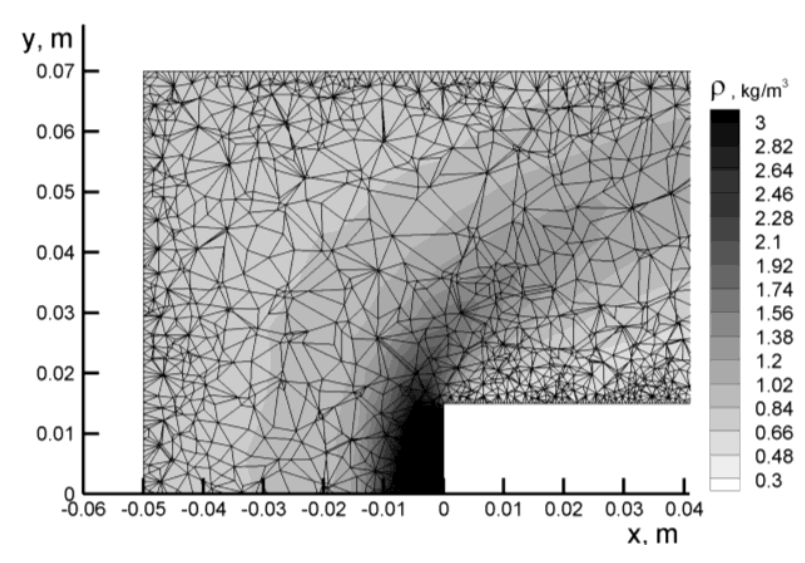

Рис. 7. Нерегулярная сетка (3)

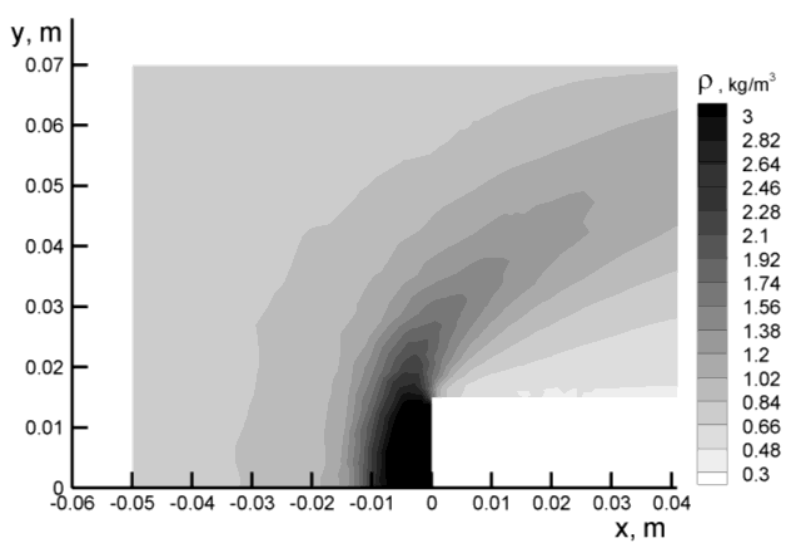

Рис. 9. Нерегулярная сетка (3)

На рис. 6 и 7 показана расчетная сетка и линии уровня плотности, на рис. 8 и 9 приведены только уровни плотности.

Результаты, полученные на равномерной сетке (1) с увеличенной вязкостью, также достаточно хорошо передают общую картину течения и в целом разумно разрешают фронт ударной волны. При этом ударная волна получается несколько расширенной по сравнению с рис. 2, фронт ударной волны занимает 8 ячеек сетки. В случае нерегулярной тетраэдраль- 
ной сетки, как видно на рис. 7 и 9, область фронта ударной волны заметно расширена по сравнению с рис. 6 и 8.

Для исследования вопроса о влиянии различных способов введения искусственной вязкости на разрешение ударных волн, приведем результат расчета при следующем введении искусственной вязкости. Коэффициент дополнительной диссипации в КГД алгоритме вычислим как

$$
\tau=\mu_{0} \frac{\left(T / T_{0}\right)^{\omega}}{p \mathrm{Sc}}+\frac{\alpha h}{c},
$$

где обозначения те же, что в (9)-(12). Коэффициент сдвиговой вязкости $\mu$ определим через коэффициент дополнительной диссипации

$$
\mu=\tau p \mathrm{Sc},
$$

коэффициент теплопроводности вычислим как

$$
\kappa=\frac{\mu}{\operatorname{Pr}(\gamma-1)}
$$

коэффициент объемной вязкости определим на основе аппроксимационной формулы

$$
\varsigma=\mu\left(\frac{5}{3}-\gamma\right)
$$

Таким образом, вместо формул (9)-(12) используются (13)-(16). Такое введение искусственной вязкости в КГД алгоритм является традиционным [6], в отличие от варианта с введением искусственной вязкости как добавки во второй вязкости (12), и исключением ее из молекулярной вязкости (9). При этом настроечным параметром является только коэффициент $\alpha$.

На рис. 10 приведены линии уровня плотности, вычисленные на основе КГД алгоритма с искусственной вязкостью вида (13)-(16), при этом $\alpha=0.3$, использована равномерная сетка (1). Аналогично рис. 2 и 4, на рис. 10 слева изображена расчетная сетка, справа приведены только уровни плотности.
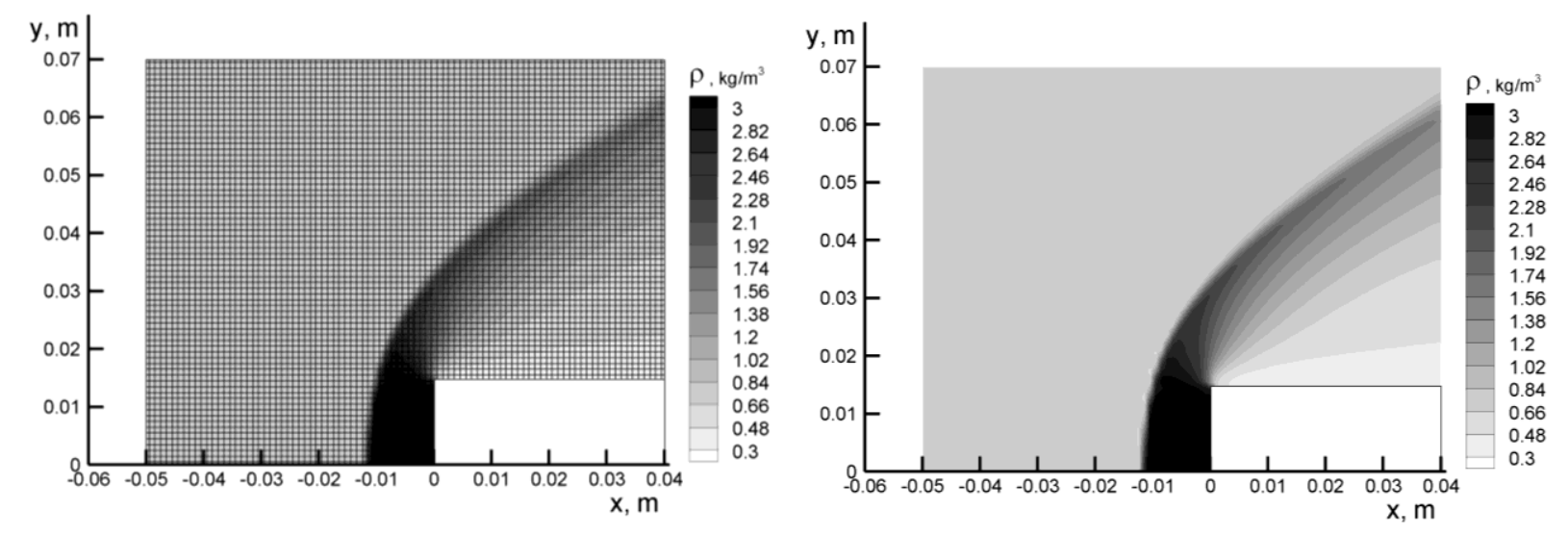

Рис. 10. Равномерная сетка (1), искусственная вязкость согласно (13)-(16)

Полученные результаты близки к результатам, полученным на равномерной сетке с искусственной вязкостью вида (9)-(12). Можно сделать вывод, что два рассмотренных способа введения искусственной вязкости являются практически равноценными при использовании одной и той же равномерной сетки. 
Для исследования влияния сгущения сетки вблизи области ударной волны были проведены расчеты на неравномерной сетке со сгущением (4). На рис. 11 приведены линии уровня плотности, вычисленные на основе КГД алгоритма с искусственной вязкостью вида (9)-(12), при этом параметры расчета те же, что соответствуют рис. 7 и 9.
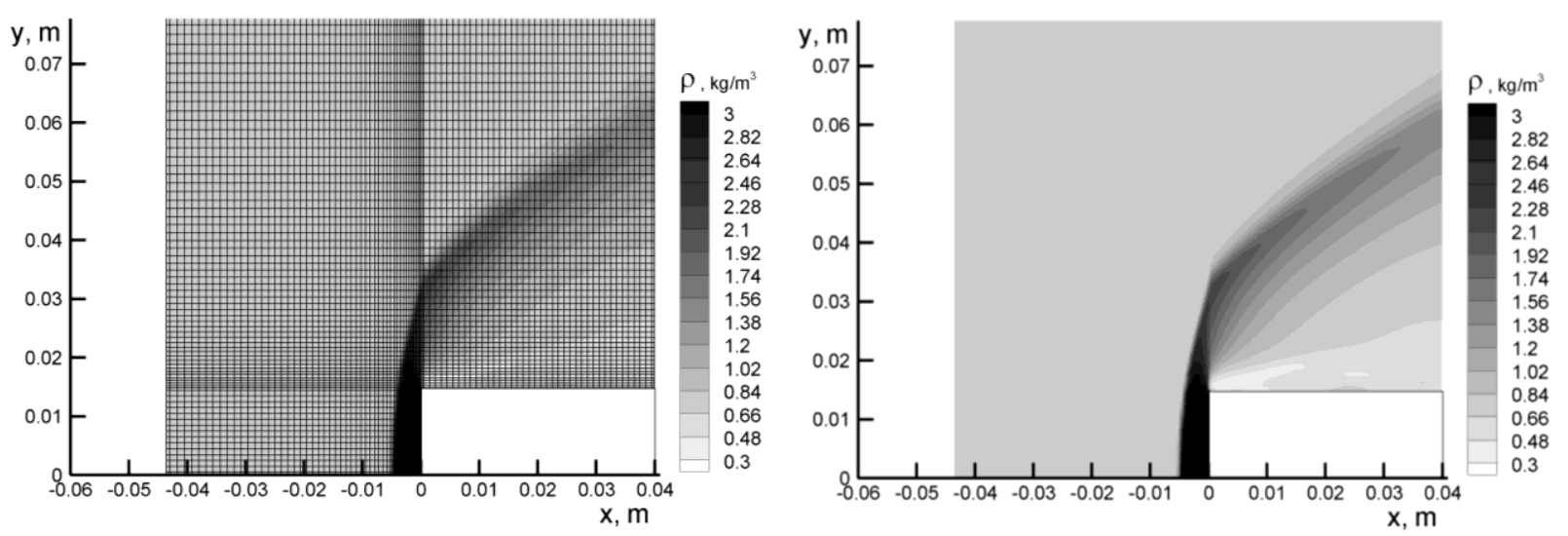

Рис. 11. Неравномерная сетка (4), искусственная вязкость согласно (9)-(12)

Аналогично рис. 2 и 4, на рис. 12 слева изображена расчетная сетка, справа приведены только уровни плотности. Хорошо видно, что область сгущения сетки сильно искажает форму фронта ударной волны. При переходе через границу области сгущения фронт испытывает излом. Кроме того, в этом случае ударная волна расположена гораздо ближе к лобовой поверхности модели, чем в случае использования равномерной и тетраэдральной сеток при тех же параметрах расчета (рис. 6-9). Отметим, что аппроксимационная формула Лунева [6] для расстояния между ударной волной и торцом обтекаемого цилиндра в данном случае дает около $0.01 \mathrm{M}$, что очень хорошо согласуется с результатами, полученными на равномерной и тетраэдральной сетках, и не согласуется с результатом, полученном на неравномерной сетке со сгущением.

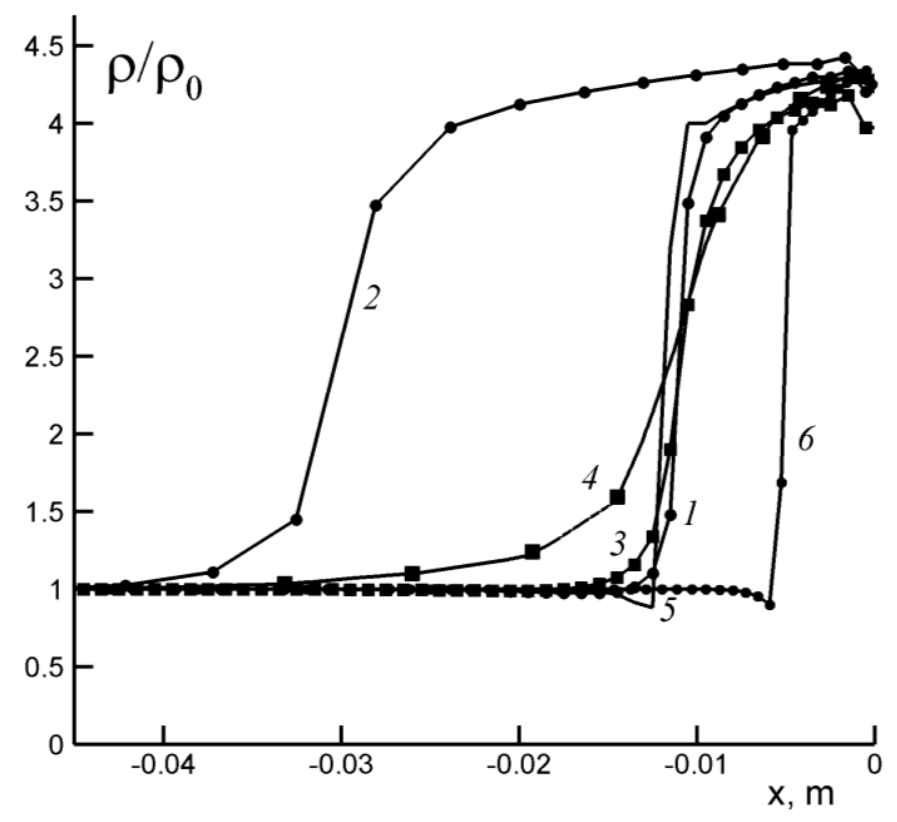

Рис. 12. Профили плотности в головной ударной волне

Также отметим, что построение сетки со сгущением в области ударной волны даже в рассматриваемом модельном случае представляет собой весьма сложную задачу, поскольку фронт ударной волны имеет криволинейную форму, и заранее расположение его неизвест- 
но. Тем более это справедливо для реальных задач, например, для расчетов обтекания летательных аппаратов различной формы, вокруг которых образуется сложная структура ударных волн, расположение которых заранее неизвестно.

Как и в случае неравномерной сетки (2) (см. рис. 2 и 4), растяжение ячеек сетки вдоль оси $Y$ (в верхней правой части рисунка) искажает форму фронта ударной волны в гораздо меньшей степени, чем в области головной ударной волны.

Разрешение фронта ударной волны на сетке можно увидеть более детально на рис. 12, на котором приведены одномерные профили плотности в зависимости от расстояния от лобовой поверхности обтекаемого тела при $y=0, z=0$. Маркеры на кривых профилей соответствуют границам ячеек сетки.

Кривые 1, 3 и 5 соответствует равномерной сетке (1), кривая 2 неравномерной сетке (2), кривая 4 нерегулярной тетраэдральной сетке (3), кривая 6 неравномерной сетке (4). Кривые 1 и 2 получены при $\delta_{1}=0.5, \delta_{2}=1$. Кривые 3,4 и 6 соответствуют $\delta_{1}=2$ и $\delta_{2}=5$. Для кривых 1-4 и 6 коэффициент $\alpha=1$. Кривая 5 получена при использовании формул искусственной вязкости (13)-(16) вместо (9)-(12), при этом коэффициент $\alpha=0.3$.

При сравнении кривых 1 и 2, полученных на равномерной и неравномерной сетках при прочих равных условиях, хорошо видно, что головная ударная волна "растягивается" вместе с ячейками сетки. Сравнение кривых 3 и 4, полученных на равномерной и нерегулярной сетках при прочих равных условиях, показывает, что вблизи поверхности обтекаемого

тела

( $x>-0.01$ м) обе сетки дают близкие результаты, поскольку при этом нерегулярная сетка достаточно подробна. При удалении от поверхности $(x<-0.01 \mathrm{~m})$ видно расширение фронта ударной волны при использовании нерегулярной сетки (кривая 4). Как и в случае регулярной неравномерной сетки, растяжение фронта ударной волны соответствует увеличению размеров ячеек сетки. Кривая 6, полученная на неравномерной сетке (4), показывает сильный сдвиг фронта ударной волны к лобовой поверхности модели, хотя фронт ударной волны разрешается очень хорошо. При использовании равномерной сетки (кривые 1,3 и 5) получаются достаточно близкие результаты при различных вариантах введения искусственной вязкости. Однако при этом заметно, что при уменьшении искусственной вязкости фронт ударной волны получается более узким.

Анализ полученных результатов приводит к выводу, что использование равномерных сеток для задач моделирования ударных волн является предпочтительным, поскольку гарантирует отсутствие искажений и сдвига фронта ударной волны.

Для количественной оценки точности результатов моделирования в табл. 1 приведено сравнение нормированных максимальных значений плотности и давления за ударной волной вблизи лобовой части модели в зависимости от используемой сетки и способа введения искусственной диссипации. Номер варианта соответствует нумерации кривых на рис. 12. Теоретические значения параметров торможения, которые используются как референсные значения (приведены в последней строке табл. 1), вычисляются для невязкого нетеплопровод-ного газа на основе условий Ренкина - Гюгонио и теоремы Бернулли [6].

Те же данные изображены на рис. 13, при этом на оси абсцисс указаны номера вариантов, соответствующих табл. 1. Видно, что данные расчетов, полученные на равномерной сетке, в целом ближе к теоретическим значениям параметров торможения.

Приведенные результаты показывают, что использование равномерной регулярной сетки при моделировании течений, включающих ударные волны, является предпочтительным, поскольку при этом увеличивается точность моделирования и разрешение фронтов ударных волн. 


\section{Таблиия 1}

\section{Максимальные значения плотности и давления в сравнении с параметрами торможения}

\begin{tabular}{|c|c|c|c|c|c|c|}
\hline & Сетка & $\begin{array}{c}\text { Искусственная } \\
\text { вязкость (формулы) }\end{array}$ & $\begin{array}{c}\text { Значения } \\
\text { коэффициентов }\end{array}$ & $\frac{\rho_{\max }}{\rho_{0}}$ & $\frac{p_{\max }}{p_{0}}$ & $\frac{T_{\max }}{T_{0}}$ \\
\hline 1 & Равномерная (1) & $(9)-(12)$ & $\begin{array}{c}\alpha=1 \\
\delta_{1}=0.5, \delta_{2}=1\end{array}$ & 4.33 & 11.98 & 2.81 \\
\hline 2 & Неравномерная (2) & $(9)-(12)$ & $\begin{array}{c}\alpha=1 \\
\delta_{1}=0.5, \delta_{2}=1\end{array}$ & 4.43 & 12.27 & 2.83 \\
\hline 3 & Равномерная (1) & (9)-(12) & $\begin{array}{c}\alpha=1 \\
\delta_{1}=2, \quad \delta_{2}=5\end{array}$ & 4.18 & 11.52 & 2.85 \\
\hline 4 & Нерегулярная (3) & $(9)-(12)$ & $\begin{array}{c}\alpha=1 \\
\delta_{1}=2, \delta_{2}=5\end{array}$ & 4.37 & 14.02 & 3.28 \\
\hline 5 & Равномерная (1) & $(13)-(16)$ & $\alpha=3$ & 4.31 & 12.08 & 2.80 \\
\hline \multirow[t]{2}{*}{6} & Неравномерная (4) & $(9)-(12)$ & $\begin{array}{c}\alpha=1 \\
\delta_{1}=2, \quad \delta_{2}=5\end{array}$ & 4.38 & 12.14 & 2.80 \\
\hline & \multicolumn{3}{|c|}{ Значения параметров торможения } & 3.42 & 11.49 & 4.00 \\
\hline
\end{tabular}
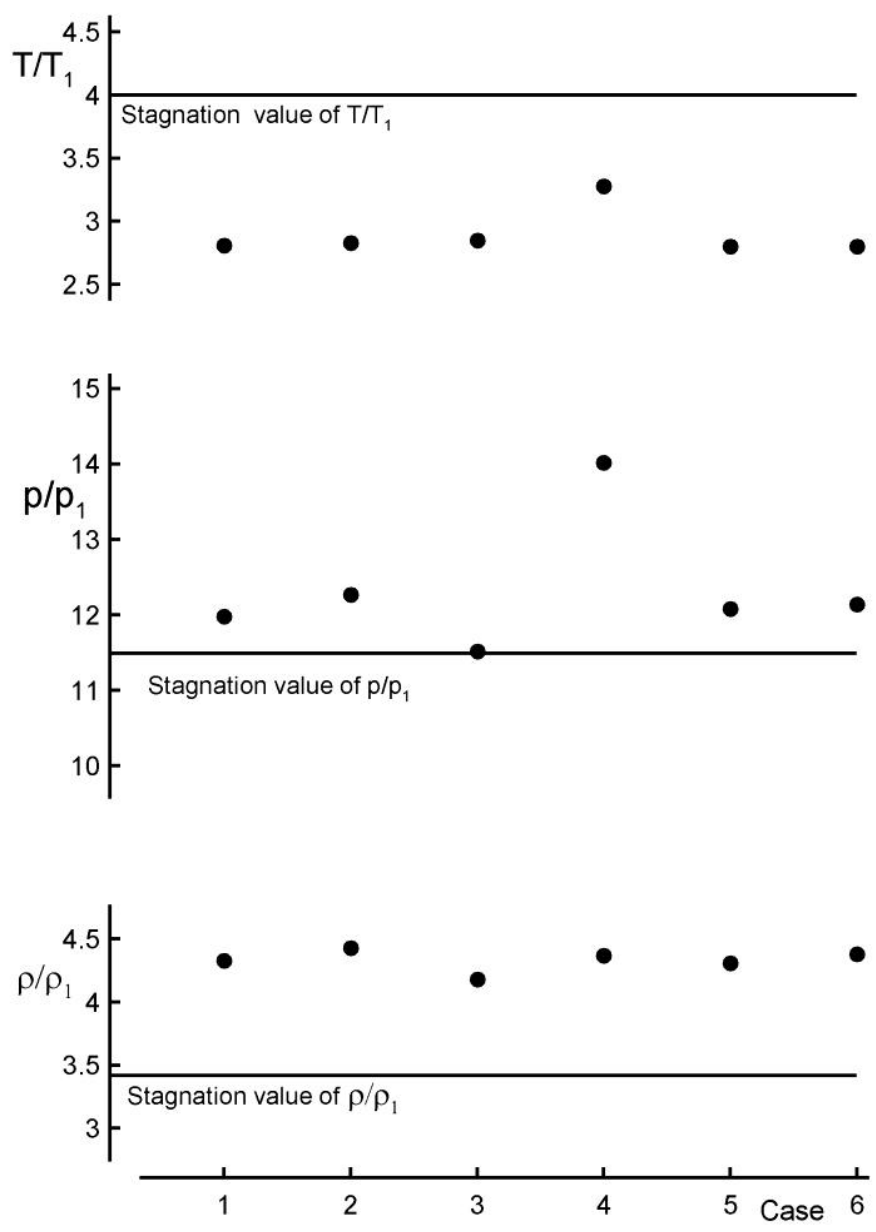

Рис. 13. Максимальные значения плотности и давления в сравнении с параметрами торможения 


\section{6. Замечания и выводы}

В работе демонстрируется влияние структуры трехмерной пространственной сетки на качество численного решения в задаче об обтекании торцевой части прямоугольного параллелепипеда потоком вязкого сжимаемого газа при числе Маха Мa $=3$. Из полученных результатов следует, что структура сетки оказывает определяющее влияние на форму и интенсивность головной ударной волны, которое может превосходить влияние других сеточных эффектов, например, величину и способ введения искусственной диссипации. Порядок точности самой разностной схемы также может оказываться несущественным при расчете разрывных решений на сильно неравномерных сетках. Поэтому вопросы построения «хороших» сеток для решения задач обтекания моделей приобретают определяющее значение.

Работа выполнена при финансовой поддержке Российского научного фонда, проект 19-11-00169.

\section{Литература}

1. Железнякова А.Л., Суржиков С.Т. Применение метода расщепления по физическим процессам для расчета гиперзвукового обтекания пространственной модели летательного аппарата сложной формы // Теплофизика высоких температур. 2013. Т. 51. № 6. С. 897-911.

2. Железнякова А.Л., Суржиков С.Т. На пути к созданию модели виртуального ГЛА. І. - М.: ИПМех РАН, 2013. $160 \mathrm{c}$.

3. Железнякова А.Л., Суржиков С.Т. Построение пространственных неструктурированных сеток на NURBS-поверхностях сложных изделий авиационной и ракетно-космической техники методом молекулярной динамики // Физико-химическая кинетика в газовой динамике. 2014. T.15, вып. 1. http://chemphys.edu.ru/issues/2014-15-1/articles/108/12/

4. Houtman E.M., Bannink W.J., Timmerman B.H. Experimental and Computational Study of a Blunt Cylinder-Flare Model in High Supersonic Flow // Delft: Delft University of Technology. 1995. Report LR-796.

5. Chetverushkin B.N. Kinetic Schemes and Quasi-Gas Dynamic System of Equations. Barselona: CIMNE. 2008.

6. Elizarova T.G. Quasi-Gas Dynamic Equations. Dordrecht: Springer. 2009. IBSN 978-3-642-0029-5.

7. Шеретов Ю.В. Регуляризованные уравнения гидродинамики. Тверь: Тверской государственный университет. 2016. 222 с. Yu. V. Sheretov. Regularized Hydrodynamic Equations, Tver State University, 2016, 222 pp. elibrary.ru/item.asp?id=30097584 (in Russian).

8. Елизарова Т.Г., Широков И.А. Регуляризованные уравнения и примеры их использования при моделировании газодинамических течений. М.:-МАКС Пресс. 2017. 136 с. https://elibrary.ru/item.asp?id=29352202 T.G. Elizarova, I.A. Shirokov. Regularized equations and examples of their use in the modeling of gas-dynamic flows.

9. Широков И.А., Елизарова Т.Г. Компьютерное моделирование обтекания модели сверхзвуковым потоком вязкого сжимаемого газа на основе квазигазодинамического алгоритма // Физико-химическая кинетика в газовой динамике. 2017. T. 18, вып. 2. http://chemphys.edu.ru/issues/2017-18-2/articles/721/ I. A. Shirokov, T. G. Elizarova. Computer simulation of the supersonic flow of a viscous compressible gas around a model body on the basis of the quasi-gas-dynamic algorithm. // Physical-Chemical Kinetics in Gas Dynamics. 2017. Vol. 18, iss. 2. http://chemphys.edu.ru/issues/2017-18-2/articles/721

10. Elizarova T.G., Shirokov I.A. Artificial dissipation coefficients in regularized equations of supersonic aerodynamics // Doklady Mathematics. 2018. Vol. 98, No 3. P. 648-651. 
11. Туник Ю.В. Проблемы численного моделирования на основе некоторых модификаций схемы Годунова // Физико-химическая кинетика в газовой динамике 2018. Т. 19, вып. 1. http://chemphys.edu.ru/issues/2018-19-1/articles/701/

12. TetGen: A quality tetrahedral mesh generator. http://tetgen.berlios.de/

13. Кудряшова Т.А., Поляков С.В., Свердлин А.А. Расчет параметров течения газа вокруг спускаемого аппарата // Математическое моделирование. 2008. 20:7. С. 13-22. Т. A. Kudryashova, S. V. Polyakov, A. A. Sverdlin. Calculation of gas flow parameters around reentry vehicle // Matem. Mod. 2008. 20:7. P. 13-22; Math. Models Comput. Simul. 2009. 1:4. P. 445-452.

14. K-100 System, Keldysh Institute of Applied Mathematics RAS, Moscow; Available at http://www.kiam.ru/MVS/resourses/k100.htm

Статья поступила в редакцию 26 июня 2019 г. 